\title{
How effective is therapeutic ultrasound in the treatment of heel pain?
}

\author{
Fay Crawford, Michael Snaith
}

\begin{abstract}
Objectives-To evaluate the therapeutic effect from ultrasound in the treatment of plantar heel pain by physiotherapists and podiatrists, and to quantify the placebo effect of this electrophysical agent. Methods-Patients experiencing episodes of plantar heel pain were allocated randomly, at each episode, to receive either true ultrasound (machine calibrated to deliver a dose of ultrasound at $0.5 \mathrm{w} / \mathrm{cm}^{2}$, $3 \mathrm{MHz}$, pulsed 1:4), for eight minutes, or sham ultrasound (only the timer on the machine activated). Each episode was treated, according to randomisation, eight times. An independent observer set the equipment before obscuring the control panel with a drape. All treatments were undertaken by the same operator. Patients' pain scores were measured on a $10 \mathrm{~cm}$ linear analogue scale before the course of eight treatments commenced and at the end of the course, and analysed using a Wilcoxon Signed-Ranks test.

Results-Nineteen patients experienced episodes of heel pain (seven bilateral). Both groups showed a reduction in pain; the improvement was $30 \%$ in the treated group and $25 \%$ in the placebo group $(\mathbf{p}=0 \cdot 5)$.

Conclusions-Therapeutic ultrasound at a dosage of $0.5 \mathrm{w} / \mathrm{cm}^{2}, 3 \mathrm{MHz}$, pulsed $1: 4$, for eight minutes is no more effective than placebo in the treatment of plantar heel pain.
\end{abstract}

(Ann Rheum Dis 1996; 55: 265-267)

Therapeutic ultrasound is used routinely by podiatrists and physiotherapists, and is prescribed by physicians in their treatment of plantar fasciitis and plantar heel pain. ${ }^{1-3}$ Although there is much literature detailing the cellular and physiological benefits of ultrasound, ${ }^{4-14}$ the few evaluative clinical trials have produced conflicting conclusions as to the effectiveness of high frequency sound waves as a treatment for painful conditions in other parts of the body. ${ }^{15-18}$ Studies which have considered the efficacy of ultrasound as a treatment for plantar heel pain lacked control groups and reached conflicting conclusions. ${ }^{12}{ }^{19}$ This type of treatment is time consuming: one complete course of ultrasound can take approximately two hours to administer. As therapists report variable results when treating painful heels, there was a clear need to evaluate ultrasound treatment, by means of a double blind trial aiming to establish if treatment is more effective than placebo. This study recorded pain scores before and after true or sham ultrasound treatment of 26 episodes of localised heel pain unassociated with systemic inflammatory disease.

\section{Patients and methods}

Patients were selected from those attending The London Foot Hospital who were referred for the management of their heel pain by general practitioners, rheumatologists, and physiotherapists. The criterion for inclusion in the study was pain on the plantar aspect of one or both heels. Exclusion criteria were: previous treatment with ultrasound; presence of fluffy calcaneal spur on radiograph; generalised joint pain; non-specific urethritis; the use of pain control (analgesics, non-steroidal antiinflammatory drugs (NSAIDs), steroids, heel pads or orthoses/appliances in shoe(s)) at the time of recruitment; a diagnosis of a seropositive or seronegative arthropathy.

Patients were requested to avoid the use of pain controlling methods for the duration of the trial in order to reduce the therapeutic effects from substances other than ultrasound. Patients' feet were examined for the presence of a positive Helbing's sign (lateral bowing of the tendo Achillis), which is a soft tissue indicator of excessive $\left(>4^{\circ}\right)$ subtalar joint pronation. $^{20}$ Lateral foot radiographs were examined for the presence and features of calcaneal spurs (well corticated, or fluffy).

With ethics committee approval, and written consent by the patient, those experiencing episodes of heel pain were allocated randomly, to receive either true or placebo ultrasound. In patients with bilateral disease, episodes were individually randomised for treatment. 'True' ultrasound comprised ultrasound for eight minutes at an intensity of $0.5 \mathrm{w} / \mathrm{cm}^{2}, 3 \mathrm{MHz}$, and pulsed 1:4, twice weekly for four weeks; painful heels in the placebo group received a sham dose of ultrasound, only the timer on the machine being activated for eight minutes. In both groups treatments were given using a Sonacel Mutiphron Mk II ultrasound machine. All treatments were given using a coupling gel.

Pain was assessed using a $10 \mathrm{~cm}$ horizontal visual analogue scale at the first and last visit. The patients' final pain score(s) were made without reference to their initial pain scores. An independent observer (SDJ) set the dosage on

Correspondence to: Ms F Crawford. 
the machine in accordance with the randomly assigned treatment group, before covering the dials with a drape to obscure them from both the operator and the patient. Treatment was given and pain scores recorded by one operator blinded to the treatment group (true or sham) throughout the trial (FC). The ultrasound machine was regularly checked during the trial by the medical physics department of The Middlesex Hospital, to ensure it was calibrated correctly. A power equation indicated that 26 results would detect an improvement of $50 \%$ or greater. Taking $20 \%$ as a placebo response, such numbers would therefore detect an improvement of $30 \%$ over placebo. Statistics were performed using Wilcoxon signed-ranks test and Fisher's exact test.

\section{Results}

Nineteen patients with 26 episodes of heel pain (seven bilateral) entered the study. Of the seven patients with bilateral disease, only one received identical randomisation (placebo) to both feet. There were thus six women (three with bilateral disease episodes) and seven men (three with bilateral disease episodes) who received true ultrasound, and five women (three with bilateral episodes) and eight men (five with bilateral episodes) who received placebo treatment. In the treatment group, the median duration of pain was 12 months; in the placebo group it was 24 months. In accordance with our selection criteria, none of the 19 patients recruited was taking NSAIDs or analgesics at the commencement of the study or at any time thereafter: this is reflective of patients attending a podiatry outpatient clinic, whose use of NSAID analgesics is much less widespread than that of rheumatology outpatients. Seven patients (two with bilateral disease) had received corticosteroid injections which had not produced relief: two episodes in the true ultrasound group (one bilateral disease episode), and five episodes in the placebo group (one bilateral disease episode).

Figure 1 shows the pain scores recorded before and after each complete course of ultrasound. Both groups showed a reduction in pain: the mean pain score before treatment in the treated group was $6 \cdot 7$, which reduced to 4.5 after treatment (mean improvement $30 \%$ ); the

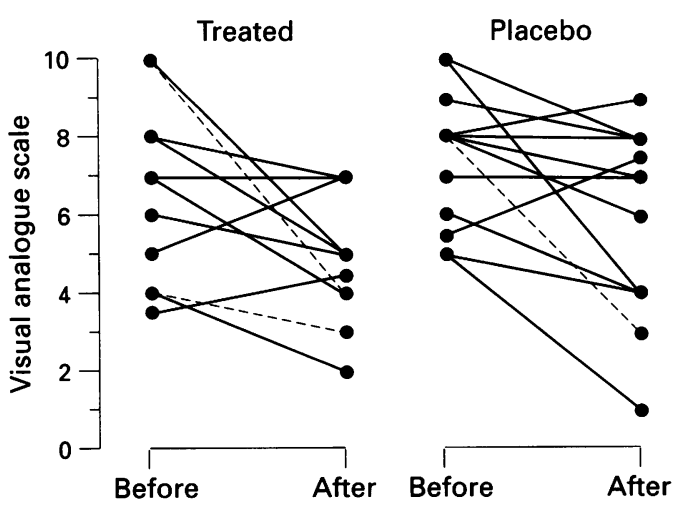

Individual pain scores before and after treatment in the treated and placebo groups. --- = Two episodes with identical pain scores. placebo group had a mean pain score of $7 \cdot 5$ before treatment, which reduced to $5 \cdot 6$ (mean improvement 25\%). This difference in improvement was not statistically significant (Wilcoxon signed-ranks test). There was no significant difference between pain duration and improvement (Fisher's exact test) in either group, and no significant difference between age of the patient and improvement (Fisher's exact test) in either group. In 21 episodes of heel pain, a positive Helbing's sign (lateral bowing of the tendo Achillis) was observed, and in 12 episodes there was radiographic evidence of a well corticated calcaneal spur; no statistical relationship existed between these two factors (Fisher's exact test). Complete resolution of pain did not occur in any episode, and pain worsened during the study in four episodes: two in the treatment group and two in the placebo group. Pain neither improved nor worsened in two episodes: one in the treatment group and one in the placebo group.

\section{Discussion}

The chronic nature of the painful heels in the trial accurately represents the population seeking treatment from The London Foot Hospital, a tertiary referral centre. Plantar fasciitis is the most common diagnosis given to pain on the plantar aspect of the heel. This term oversimplifies the painful heel, which may have one of several aetiologies, ${ }^{21}$ explanations of which are outside the remit of this paper. The aetiology of plantar heel pain is believed to be associated with lower limb biomechanics - that is, pronation producing tension on the soft tissues of the plantar surface-or part of a systemic inflammatory condition. Patients who presented with likely signs of systemic inflammatory disease related heel pain were excluded from the trial in an effort to assess the ultrasound treatment with minimal variables.

There is some debate as to whether excessive subtalar joint pronation is related to both heel pain and calcaneal spur formation. ${ }^{1922}{ }^{23}$ Our finding of no statistically significant relationship between the presence of heel spur and Helbing's sign in the patients participating in this study adds to that debate, and further work is required to define the relationship between subtalar joint pronation, heel pain, and calcaneal spur formation. We acknowledge that, while Helbing's sign may indicate excessive subtalar joint pronation, bowing of the tendo Achillis may apparently be absent when the subtalar joint is actually pronated. As yet, no error free method of measuring subtalar joint pronation is available.

The selection of the dose of ultrasound was based on the clinical judgment of the authors and reached in consultation with physiotherapy colleagues. The intensity of $0.5 \mathrm{w} / \mathrm{cm}^{2}$ and the number of treatments given are within the standard range used by podiatrists and physiotherapists. ${ }^{24}$ Intensities of $0.5-1.5 \mathrm{w} / \mathrm{cm}^{2}$ for periods of five to 10 minutes have been recommended for the treatment of foot conditions. ${ }^{3}$ The number of treatments given is also within the standard treatment range. 
Guidelines for the selection of ultrasound doses are often vague and are based on empirical evaluation and in vitro models, rather than in vivo quantitative studies.

One study ${ }^{1}$ evaluating the use of ultrasound in the treatment of plantar fasciitis stated the optimum intensity to be $2-3 \mathrm{w} / \mathrm{cm}^{2}$ but, in common with physiotherapy texts, ${ }^{24}$ offered no justifying explanation of the rationale behind this choice of intensity. There is a need for therapists to prove the efficacy of different dosages of ultrasound across the therapeutic range, considering different parameters such as pulsing versus continuous beam, intensity, frequency, and probe movement. These different dose parameters each need to be evaluated with statistically appropriate and controlled populations of patients, in order to substantiate results. Our findings demonstrate that, though there was a slightly greater mean improvement in heel pain in the treatment group, it was of no statistical significance. It is possible that randomised, double blind trials using ultrasound doses of differing parameters to treat systemic inflammatory disease related heel pain, or acute heel pain, would produce different conclusions as to the effectiveness of this treatment. Further double blind studies are required to make a quantitative assessment of the therapeutic benefit of ultrasound.

What is clear from our results is that the placebo effect of ultrasound in this trial was $25 \%$. While the number of episodes measured in this study may have resulted in a type II error, we do not consider small reductions in heel pain to be clinically meaningful. The study had a $90 \%$ chance of detecting an improvement of $50 \%$ or greater in the treatment group, including the placebo response. Most studies evaluating treatment efficacy would only consider improvements of $>50 \%$ to be clinically meaningful. ${ }^{25}$ Using this criterion, it is important to note that, from a sample of 26 episodes of pain, such an improvement was observed in only eight episodes (fig 1), four of which occurred in the placebo group. It therefore appears that ultrasound at this intensity is of no clinical value in the treatment of localised heel pain.

The authors wish to acknowledge the help and assistance received from the late Scott $\mathrm{D}$ Jenkins, teacher at The London
Foot Hospital from 1986 to 1992, who acted as the independent observer during the trial, and Ms D Price, Medical Statistics Department The Hammersmith Department, The Hammersmith Hospital London, for her statistical analysis of the results. We also thank Dr Madeleine
Rooney for her valuable contribution to the discussion of these results.

1 Whiting M F. The treatment of painful conditions affecting the heel and plantar fascia. Chiropodist 1975; 30: 247-63. 2 Clark G R, Stenner L. Use of therapeutic ultrasound. Physiotherapy 1976; 62: 185-90.

3 Bruno J, Hefland A. Ultrasound and some podiatric considerations. F Am Podiatr Med Assoc 1976; 66: 682-6.

4 Windsor A M. Introduction to therapeutic ultrasound. Chiropodist 1979; 34: 282-7.

5 McDiarmid T, Burns $\mathbf{P}$ N. Clinical application of therapeutic ultrasound. Physiotherapy 1987; 73: 155-62.

6 Deutic ultrasound. Physiotherapy 1987, 73: 155-62. Physiotherapy 1987; 73: 116-20.

7 Chapman I V, MacNally N A, Tucker S. Ultrasound changes in rates of influx and efflux of potassium ions in rat thymocytes in vitro. Ultrasound Med Biol 1979; 6; 47-58.

8 Lehmann J F. The biophysical mode of action of biologic and therapeutic ultrasonic reactions. $\mathcal{f}$ Acoust Soc Am 1953; 25: 17-25.

9 Wyper D J, McNiven D R, Donnelly T J. Therapeutic ultrasound and muscle blood flow. Physiotherapy 1978; 54: 321-22.

10 Lehmann J F, Biegler R. Changes in potentials and temperature gradients in membranes caused by
ultrasound. Arch Phys Med Rehabil 1954; 35: 287-93.

11 Madsen P W, Gersden J W. The effect of ultrasound on the conduction velocity of peripheral nerve. Arch Phys Med Conduction velocity of perit

12 Maxwell L. Therapeutic ultrasound: its effects on the cellular and molecular mechanisms of inflammation and cellular and molecular mechanisms of
repair. Physiotherapy 1991; 78: 421-6.

13 Szumski A J. Mechanisms of pain relief as a result of therapeutic application of ultrasound. Phys Ther Rev 1959; 40: 16-9.

14 Chapman I V. The effect of ultrasound on the potassium content of rat thymocytes in vitro. Radiol 1974; 47: 411-5.

15 Swan Downing D, Weinstein A. Ultrasound therapy of subacromial bursitis. Phys Ther 1986; 66: 194-9.

16 Grynbaum B B. An evaluation of the clinical use of ultrasonics. Am f Phys Med 1954; 53: 75-8.

17 Binder A, Hodge G, Greenwood A M, Hazleman B L, Page Thomas D. Is therapeutic ultrasound effective in Page Thomas D. Is therapeutic ultrasound effective

18 Meuller E E, Sedgewick M, Schulz B S, Vaden M R. A placebQ-controlled study of ultrasound treatment for periarthritis: symposium on ultrasonics. Am f Phys Med 1953; 33: 31-5.

19 Snook G A, Chrisman D O. The management of subcalcaneal pain. Clin Orthop 1972; 82: 163-8.

20 Neale D, Rendal G, Boyd P. The adult foot. In: Lorimer D, ed. Neale's common foot disorders, diagnosis and management: a general clinical guide, 4th edn. Edinburgh: Churchill Livingstone, 1993; 61.

21 Jahss M H. Disorders of the foot and ankle. vol 2, ch 47. Philadelphia: W B Saunders Company, 1991; 1390-2.

22 Weiner B E, Ross A S, Bogan R J. Biomechanical heel pain: a case study. F Am Podiatr Assoc 1979; 69: 723-6.

23 Shama S S, Kominsky S J, Lemont H. Prevalence of non painful heel spur and its relations to postural foot position. 7 Am Podiatr Assoc 1983; 73: 122-3.

24 Forster A, Palastanga N, eds. Ultrasonic therapy. In: Clayton's electrotherapy, theory and practice, 9th edn, chapter 5. Eastbourne: Bailliere Tindall, 1987; 177-9.

25 Ward J R, Williams H J, Egger M J, et al. Comparison of auranofin, gold sodium thiomalate, and placebo in the treatment of rheumatoid arthritis: a controlled trial. Arthritis Rheum 1983; 26: 1303-15. 